\title{
OPEN CRISPR/Cas9-mediated knockout of Mct8 reveals a functional involvement of Mct8 in testis and sperm development in a rat
}

\author{
Hee Sook Bae ${ }^{1,4}$, Yun-Kyeong Jin ${ }^{2,4}$, Sangwoo Ham ${ }^{1}$, Hee Kyoung Kim ${ }^{2}$, Hyejung Shin ${ }^{1}$, \\ Gyu-bon Cho ${ }^{1}$, Kyu Jun Lee ${ }^{1}$, Hohyeon Lee ${ }^{1}$, Kyeong-Min Kim², Ok-Jae Koo ${ }^{1}$, Goo Jang ${ }^{2}{ }^{2}$, \\ Jung Min Lee ${ }^{1,3}$ \& Jae Young Lee ${ }^{1 \bowtie}$
}

Thyroid hormone (TH) has long been believed to play a minor role in male reproduction. However, evidences from experimental model of thyrotoxicosis or hypothyroidism suggests its role in spermatogenesis. Cellular action of TH requires membrane transport via specific transporters such as monocarboxylate transporter 8 (MCT8). SLC16A2 (encodes for MCT8) inactivating mutation in humans can lead to Allan-Herndon Dudley-syndrome, a X-linked psychomotor and growth retardation. These patients present cryptorchidism which suggests a role of MCT8 during spermatogenesis. In this study, we found that Mct8 is highly expressed during early postnatal development and decreases its expression in the adulthood of testis of wild-type male rats. Histological analysis revealed that spermatogonia largely lacks MCT8 expression while spermatocytes and maturing spermatids highly express MCT8. To further understand the role of Mct8 during spermatogenesis, we generated Slc16a2 (encodes MCT8) knockout rats using CRISPR/Cas9. Serum THs (T3 and T4) level were significantly altered in Slc16a2 knockout rats when compared to wild-type littermates during early to late postnatal development. Unlike Slc16a2 knockout mice, Slc16a2 knockout rats showed growth delay during early to late postnatal development. In adult SIc16a2 knockout rats, we observed reduced sperm motility and viability. Collectively, our data unveil a functional involvement of MCT8 in spermatogenesis, underscoring the importance of $\mathrm{TH}$ signaling and action during spermatogenesis.

Thyroid hormones (THs) are well known to play a crucial role in the development of the central nervous system and in the regulation of cellular differentiation and metabolism ${ }^{1,2}$. Both prohormone, thyroxine (T4) and relatively minimal amount of genomically active tri-iodothyronine (T3) secreted from thyroid gland can be actively transported into the cells via specific transporters expressed at the plasma membrane ${ }^{1}$. These include monocarboxylate transporter 8 and 10 (MCT8 and MCT10), organic anion-transporting polypeptide 1c1 (OATP1C1) and L-type amino acid transporter 1 and 2 (LAT1 and LAT2) ${ }^{3}$. All of these transporters transport aromatic amino acids except MCT8 which only transports THs, positioning it as the most specific TH transporter ${ }^{4}$.

Male reproductive organs such as testis and epididymis were long thought to be unresponsive organs for THs. However increasing evidences suggest for their potential role in these organs.

Molecular machineries that can exert cellular TH entry to its genomic action were all shown to be expressed in testis ${ }^{5}$. For instance, specific nuclear receptors for THs which is required to exert gene transcription mediated by THs are shown to be expressed in both rodent and human testis ${ }^{6,7}$. Furthermore, TH transporters and deiodinases were shown to be expressed in testis 5 .

The potential impact of TH in male reproductive organs development is manifested in animal models of hypo- or hyperthyroidism which resulted in substantial alteration in physiology of testis development ${ }^{8}$. TH action is not only dependent on the serum concentrations of the hormones, but also on tissue-specific cellular TH transporters, which can regulate local TH availability and elicit cell-specific developmental events. Human loss-of-function mutation of MCT8, Allan-Herndon-Dudley syndrome (AHDS) shows cryptorchidism

\footnotetext{
${ }^{1}$ ToolGen, Inc., \#1204, Byucksan Digital Valley 6-cha, Seoul, South Korea. ${ }^{2}$ Laboratory of Theriogenology, Department of Veterinary Clinical Science, College of Veterinary Medicine, Seoul National University, Seoul, South Korea. ${ }^{3}$ School of Life Science, Handong Global University, Pohang 37554, South Korea. ${ }^{4}$ These authors contributed equally: Hee Sook Bae and Yun-Kyeong Jin. ${ }^{\varpi}$ email: jy.lee2@toolgen.com
} 
A

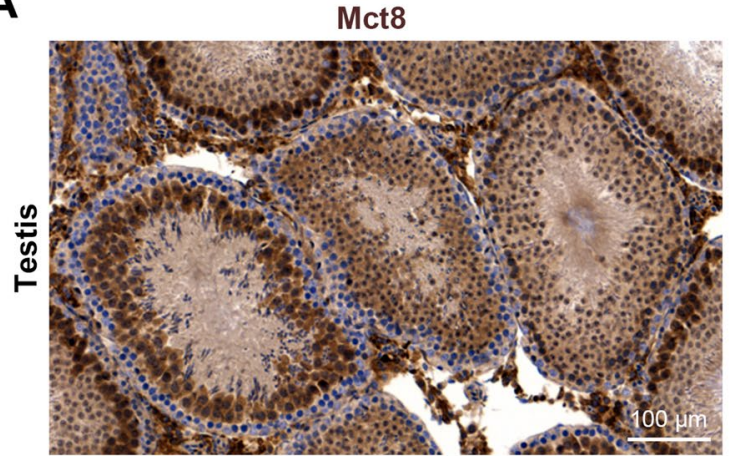

B

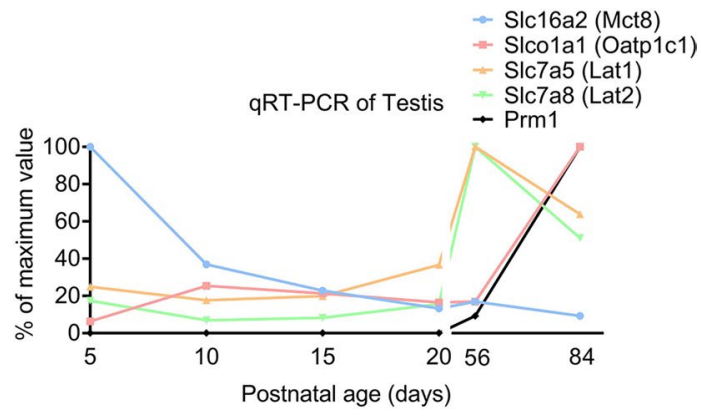

Figure 1. TH transporters expression in the rat testis during its development. (A) Histological examination of Mct8 expression in the wild-type rat testis at postnatal day 56. Note that the Mct8 is absent in immature germ cells (spermatogonium) whereas it is expressed in maturing germ cells (spermatocytes). (B) Gene expression analysis of TH transporters in rat testis during development. The data are expressed relative to highest expression of each genes tested. $n=3$.

(undescended testes), demonstrating functional significance of TH transporter in testicular development ${ }^{9,10}$. In an attempt to recapitulate phenotypes related to AHDS, mice lacking Mct8 were generated ${ }^{11,12}$. Although, serum TH hormone levels were found to be altered as observed in AHDS patients, no phenotypes related to abnormal testis development were reported ${ }^{11,12}$.

Because rat is more physiologically relevant to human than mouse $\mathrm{e}^{13}$ and represented phenotypes of human which are not observed in the mouse counterpart ${ }^{14}$, we sought to develop a novel rat model of Mct8 deficiency using CRISPR/Cas9 gene-editing. Our data shows that, unlike mice lacking Mct8, mutant rat showed significant growth retardation and altered spermatogenesis, which may implicate the potential importance of proper $\mathrm{TH}$ transport for mammalian gonad development.

\section{Results}

Mct8 is expressed on rat testis. Given potential impact of TH levels on gonadal development ${ }^{5}$, we first immunostained testis section from wild-type adult male rat (p50) with anti-Mct8 and found the expression of Mct8 primarily in maturing spermatids whereas spermatogonia largely lacks its expression (Fig. 1A, Supplementray Fig. 1). Furthermore we also immunostained cauda epididymis and found Mct8 is localized to epithelium and spermatozoa (Supplementary Fig. 2). We then seek to identify the expression pattern of known TH transporters previously shown to be expressed in rodent testis ${ }^{15,16}$ (for review see ${ }^{17}$ ) throughout developmental stages. For this, we performed qRT-PCR-based gene expression analysis of transcripts of TH transporters, Slc16a2 (Mct8), Slco1a1 (Oatp1c1), Slc7a5 (Lat1) and Slc7a8 (Lat2) at postnatal day 5, 10, 15, 20, 56 and 84 (p5, p10, p15, p20, p56, p84). Prm1 (Protamine, which is known to be expressed on in the adult testis) was included as internal control. In line with previous observation in mouse ${ }^{16}$, Mct8 is highly regulated when compared to other TH transporters during early developmental stage (from p5 to p15) (Fig. 1B and Supplementary Fig. 3). As Sertoli, Leydig and Germ cell proliferation were reported to occur until p15 during rodent testis development ${ }^{5}$ and as Mct8 is the most efficient and specific TH transporter ${ }^{3}$, highly regulated expression of Mct8 during early testis development indicates the importance of $\mathrm{TH}$ action for testicular cell proliferation. However, the gene expressions for other TH transporters such as Oatp1c1, Lat1 and Lat2 were found to sharply increase during adulthood which may suggest for their role during adulthood.

Generation of Mct8 deficient (Slc16a2 -/-) rats. To investigate the potential impact of Mct8 in testicular development, we electroporated Cas9 protein and CRISPR gRNA targeting exon 1 of rat Slc16a2 (Fig. 2A) into pronuclear-stage embryos of Sprague-Dawley rats. Upon embryo transfer of electroporated embryos to pseudo-pregnant foster mothers, we obtained mutant pups that were found to have 10 base pairs deletion analysed by targeted deep sequencing (-10; Fig. 2B; Supplementary Table 1). This -10 deletion mutation in exon 1 is expected to cause premature stop codon which can induce knockout of Slc16a2 and as expected, mRNA of Slc16a2 was barely detectable in the testis of Slc16a2-/- rats (Fig. 2C). We also performed western blot analysis and further validated undetectable expression of Mct8 in Slc16a2-/- rat testis (Fig. 2D). For maintenance of the colony, we crossed Slc16a2+/+ male rats with Slc16a2 \pm female rats. The genotypes of offspring derived from this mating was consistent with Mendelian inheritance (Supplementary Table 2). To evaluate the fertility of the mutant male rats, we crossed Slc16a2-/- male rats with Slc16a2+/+ female rats and normal litter size was obtained when compared with Slc16a2+/+ male rats (Supplementary Fig. 4). To further examine fertilization ability, we performed in vitro fertilization test by inseminating the oocytes from Slc16a2+/+ rats with the sperm from Slc16a2-I- and Slc16a2+/+ rats. This revealed a lower fertilizing ability of Slc16a2-/- rat sperm (lower number of embryos developed into two-cells; Supplementary Table 3). These indicate that even though lower fertilization rate was observed in Slc16a2-/- rat sperm, once successfully, these embryos can develop normally. For further phenotypic analysis described throught this manuscript, F3 founders of Slc16a2+/+ and Slc16a2-/-were utilized. 
A

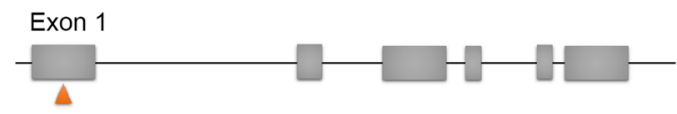

Slc16a2 gRNA:

GGTGCCTCGGGTCTCCACTGTGG
D

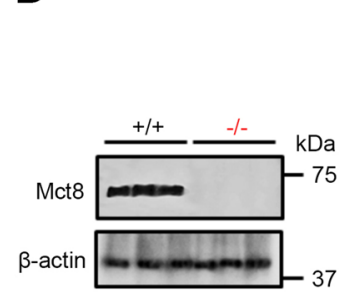

E
B

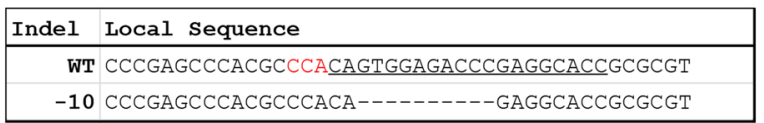

C
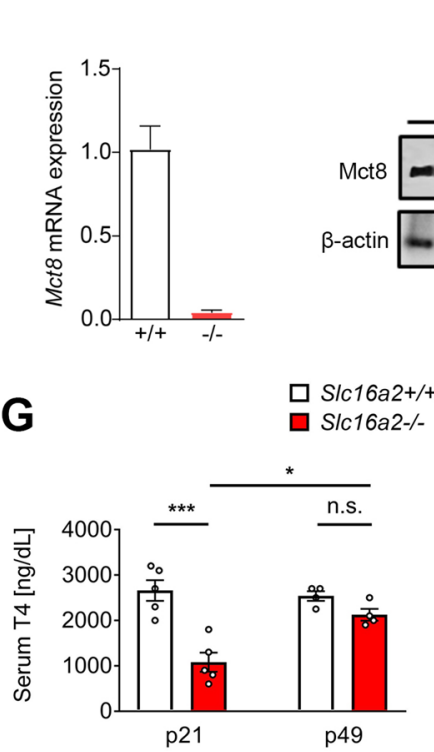

S/c16a2+/+ S/c16a2-/-

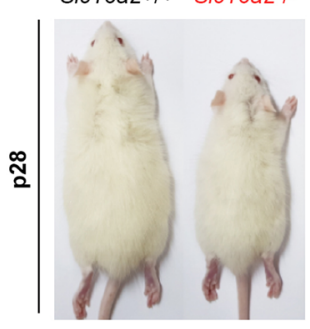

$\mathbf{F}$

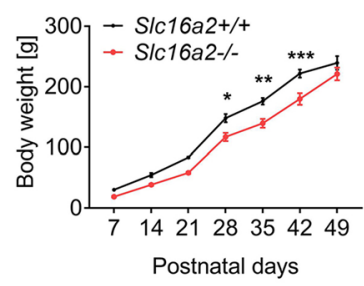

Figure 2. Transient growth retardation associated with altered serum TH levels in Slc16a2-/- rats generated using CRISPR/Cas9. (A) Schematic representation of the targeting site at rat Slc16a2 loci. Exon 1 of rat Slc16a2 was targeted using specific CRISPR gRNA shown below. (B) Targeted deep-sequencing reads of the mutant allele from F0 founder. The gRNA-targeting sequences are underlined and the PAM sequence is highlighted in red. The deletions are indicated as. (C) qRT-PCR analysis of testes of F3 founders of Slc16a2+/+ and Slc16a2-/( $n=5$ for each genotypes). (D) Immunoblot analysis of testes of F3 founders of Slc16a2+/+ and Slc16a2-l-. (E) A representative image of Slc16a2+/+ and Slc16a2-/- male rats at postnatal day 28, showing growth retardation phenotype in Slc16a2-/- male rat. (F) The growth curve of Slc16a2+/+ and Slc16a2-I- male rats showing a transient growth retardation of Slc16a2-/- from p28-42. (G) Serum T4 concentrations of Slc16a2+/+ and Slc16a2-/- male rats were determined at p21 and p49. Slc16a2-/- rats. (H) Serum T3 concentrations of Slc16a2+/+ and Slc16a2-/- male rats were determined at p21 and p49. (I) Serum T3/T4 ratio of Slc16a2+/+ and Slc16a2-/- male rats at $\mathrm{p} 21$ and $\mathrm{p} 49 . n=5, P<0.05$; $^{*}, P<0.01$; $^{* *}, P<0.001$; $^{* *}$.

Reduced growth rate along with thyroid dysfunction of Slc16a2-/-rats. Unlike Slc16a2-/mice ${ }^{12,15,18}$, Slc16a2-/- rats showed growth delay with visibly smaller body size observed from postnatal days 28 until 42 (Fig. 2E) and this difference was alleviated from postnatal day 49 (Fig. 2F). We next analyzed serum TH status of Slc16a2-/- male and their wild-type littermates at p21 and p49. In Slc16a2-/- rats, serum T4 levels were significantly decreased at p21, however, there were no significant differences between Slc16a2-/- and Slc16a2+/+ serum T4 levels at p49. (Fig. 2G). On the other hand, the serum T3 levels of Slc16a2-I- was found to be significantly increased when compared to Slc16a2+/+ rats at p21 and this difference was lessened at p49 (Fig. $2 \mathrm{H}$ ). Overall, there was 4.47 fold increase in the mean T3/T4 ratio in the Slc16a2-/- male rats at p21 which was partially normalized by 49 (1.51 fold increase in the mean serum T3/T4 ratio in Slc16a2-/- when compared to Slc16a2+/+ rats) (Fig. 2I). Thus, these results indicate that in Slc16a2-/- male rats, serum hyperthyroidism (increased serum T3 level) during p21 to p42 may have caused hypermetabolism which caused reduced growth rate of this mutant.

Increased thyroid gland follicular size in Slc16a2-/- rats. Next, we addressed the consequences of Mct8 deficiency on thyroid gland morphology. For this, we stained thyroid gland sections with H\&E from both Slc16a2+/+ and Slc16a2-/- rat males at p28. Similar to Slc16a2-/-mice, Slc16a2-/- rats showed increased thyroid gland whole follicular area (Fig. 3A,B). Furthermore, significantly increased size of follicles in thyroid gland was found in Slc16a2-/- when compared to Slc16a2+/+ male rats. This may suggest that as reported in Slc16a2-/- mice ${ }^{18,19}$, thyroidal TH concentration may have increased in Slc16a2-/- male rats which was reflected as increase in follicle size. 
A

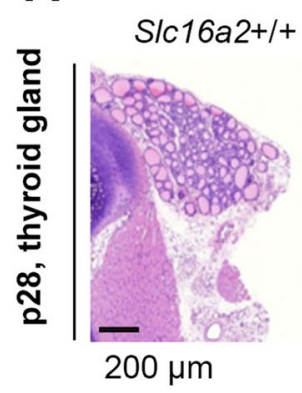

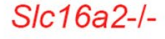

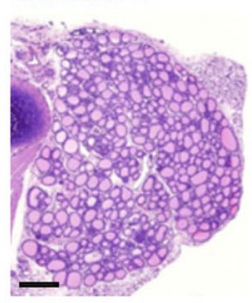

B

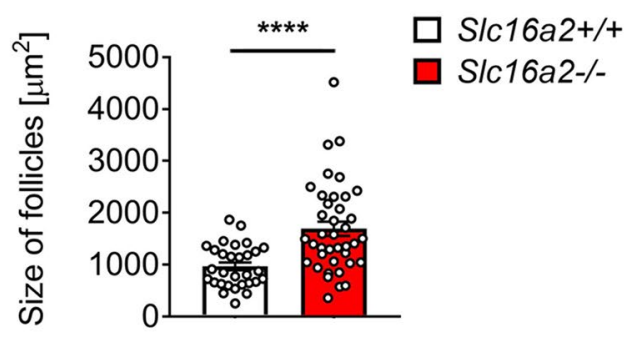

Figure 3. Histological examination of Slc16a2+/+ and Slc16a2-/- male rat thyroid glands. (A) Schematic representation of $\mathrm{H} \& \mathrm{E}$-stained sections from thyroid glands of p28 Slc16a2+/+ and Slc16a2-/- male rats. (B) Morphometric analysis of thyroid gland sections showing the size of follicle. $n=5, P<0.0001$;*** .

Testis maldevelopment in Slc16a2-I-rats. To address whether Slc16a2-/- male rats recapitulate abnormal testicular development as reported in AHDS patients, we first examined gross morphology of testis from Slc16a2+/+ and Slc16a2-/- rats. We found that both the size (Fig. 4A) and weight (Fig. 4B) of the testes of Slc16a2-/- rats were reduced when compared with Slc16a2+/+ rats at p21. We then performed histological analysis of H\&E stained sections from testes of Slc16a2-/- and Slc16a2+/+ rats (Fig. 4C). Although no obvious defects were identified, we found that Slc16a2-I- rats showed reduced diameters of round seminiferous tubules when compared with Slc16a2+/+ rats (Fig. 4D). We also performed similar analysis at p49, a timepoint when testis are fully mature during normal rat development. On the contrary to $\mathrm{p} 21$, we identified increased both the size (Fig. 4E) and weight of the testes of Slc16a2-/- rats (Fig. 4F) when compared with Slc16a2+/+ rats which is similar observation as reported in Slc16a2-/- mice ${ }^{16}$. As neonatal alteration of TH can lead to central abnormalities in the hypothalamic-pituitary-thyroid axis, we analysed serum testosterone level at $\mathrm{p} 49$. This analysis revealed a significant increase in serum testosterone level in Slc16a2-/- rats when compared to Slc16a2+/+ rats, which may have contributed to enlargement of testis size in Slc16a2-/- rats. Furthermore, as the size of testis was reported to be highly dependent on the number of Sertoli cells ${ }^{20}$, we counted the number of Sertoli cells per seminiferous tubules at p28 and p56 by immunostaining against Vimentin, a cytoplasmic Sertoli cell marker ${ }^{21}$. We found that, the number of Sertoli cells per seminiferous tubules was reduced at p28, whereas increased at p56 in Slc16a2-/- rats when compared with Slc16a2+/+ rats (Supplementary Fig. 5). These results indicates that the local hypothyroidism due to Mct8 deficiency in Slc16a2-/- rats may cause delay in Sertoli cell differentiation and prolonged proliferation phase.

Impaired spermiogenesis in Slc16a2-/-rats. To further investigate the impact of lacking Mct8 in male reproductive biology, we investigated spermiogenesis. For this, we prepared H\&E stained sections from cauda epididymis from Slc16a2+/+ and Slc16a2-/- rats. We identified abnormal spermatozoa in Slc16a2-/-rats at 449 . Furthermore, the sperm density was reduced in Slc16a2-/- when compared with Slc16a2+/+ rats at p49 (Fig. 5A and Supplementary Fig. 6). These were also found in corpus epididymis where sperm density was found reduced in Slc16a2-/- when compared with Slc16a2+/+ rats (Supplementary Fig. 6). Moreover, caudal epididymal spermatozoa imaging revealed that abnormal sperm morphology was found in Slc16a2-/- when compared with Slc16a2+/+ rats (Supplementary Fig. 7). Furthermore, epididymal epithelial cells seemed abnormal in Slc16a2-/- when compared with Slc16a2+/+ rats (Fig. 5A). To further characterize potential deficit in spermiogenesis, we performed computer-assisted semen analysis (CASA) to examine sperm concentration and motility in Slc16a2-/- rats at p49. We found that the number of spermatozoa was reduced in Slc16a2-/- rats when compared with Slc16a2+/+ rats (Fig. 5B). Furthermore, sperm motility of Slc16a2-/- rats were decreased when compared with Slc16a2+/+ rats (Fig. 5C, Supplementary Video 1 and 2). All other parameters of CASA imaging, including average path velocity (VAP), straight line velocity (VSL) and curvilinear velocity (VCL) (Fig. 5D-F).

Together, these results indicate that Mct8 deficiency in male rats can affect testis development and spermiogenesis.

\section{Discussion}

It has been well established that TH level influences testis development ${ }^{5}$. TH action in local tissues and cells can be regulated by specific TH transporters such as Mct $8^{3}$. The potential importance of Mct8 in testis development was suggested from the patients with AHDS where cryptorchidism ${ }^{4,9}$. Furthermore, we have found that Mct8 is expressed throughout early to late postnatal development as a dominant $\mathrm{TH}$ transporter, indicating potential impact of this transporter in testis development (Fig. 1). Interestingly, developmental expression pattern of Slc16a2 is somewhat in line with mouse developmental expression pattern of Thra, a nuclear TH receptor highly expressed in Sertoli cells ${ }^{5}$. One can expect a potential important role of Mct8 in TH transport in Sertoli cells.

To study the potential impact of Mct8 deficiency in testis development, we generated the rat model of Mct8 deficiency utilizing CRISPR/Cas9 technology (Fig. 2A,B). As genome and physiology of rats has been shown to be closer to humans ${ }^{22-24}$, we selected rats rather than mice to study the role of Mct8 in testis development. In fact, 
A

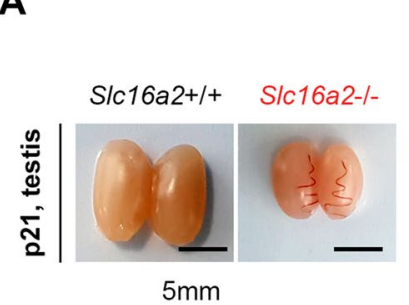

$\mathbf{E}$

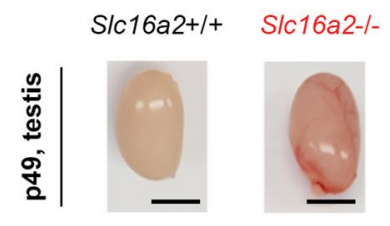

B

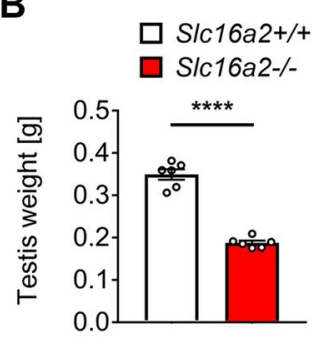

$\mathbf{F}$

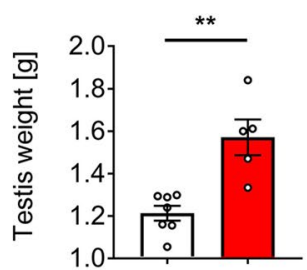

I

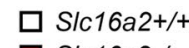

Slc16a2-/-

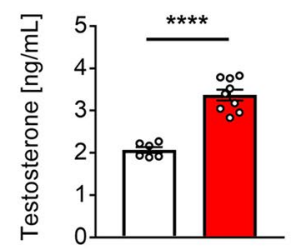

C

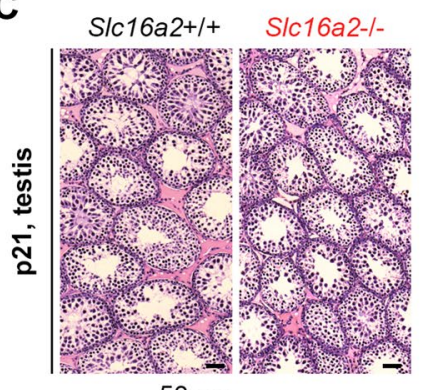

$50 \mu \mathrm{m}$

G

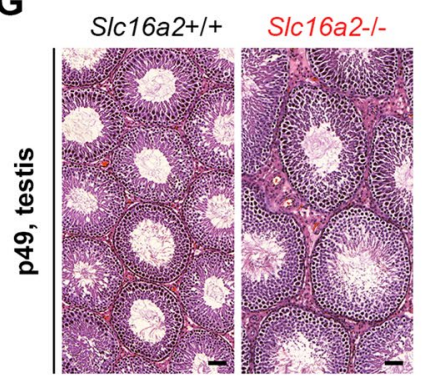

H
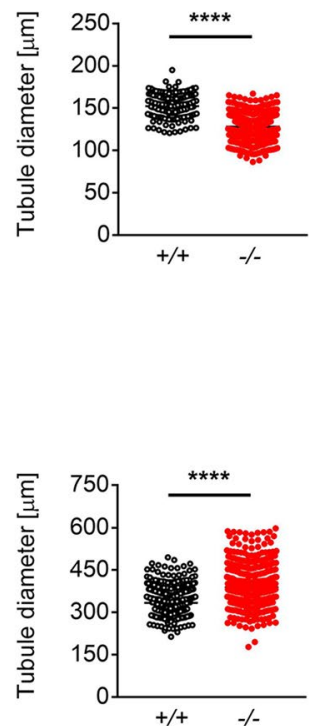

Figure 4. Altered testis development of Slc16a2-/- rat. (A) Representative images of Slc16a2+/+ and Slc16a2-/- rats at p21. (B) Testis weights of Slc16a2+/+ and Slc16a2-/- rats at p21. $n=6$ for each genotypes. (C) Representative images of H\&E stained sections of Slc16a2+/+ and Slc16a2-/- rats at p21. (D) Diameter of seminiferous tubules of Slc16a2+/+ and Slc16a2-/- rats at p21 $n=4$ for each genotypes. (E) Representative image of Slc16a2+/+ and Slc16a2-/- rats at p49. (F) Testis weights of Slc16a2+/+ and Slc16a2-/- rats at p49. $n=7$ for Slc16a2+/+ and $n=5$ for Slc16a2-/-. (G) Representative images of H\&E stained testis sections of Slc16a2+/+ and Slc16a2-/- rats at p49. (H) Diameter of seminiferous tubules of Slc16a2+/+ and Slc16a2-/- rats at $\mathrm{p} 49 . n=4$ for each genotypes. (I) Serum testosterone levels of Slc16a2+/+ and Slc16a2-/- male rats at p49. $n=6$ for Slc16a2+/+ and $n=9$ for Slc16a2-/-, $P<0.01 ;^{* *}, P<0.001 ;^{* * *} P<0.0001 ;^{* * * *}$.

we found a transient growth delay in Slc16a2-/- rats (Fig. 2D,E) which was not found in Slc16a2-/- mice ${ }^{11,12}$. Slow growth rate during early to late postnatal development (p21-42) in Slc16a2-/- rats seemed to be associated with serum hyperthyroidism as during these timepoints, we found a significant increase in serum T3 level at early postnatal development (p21). This increased level of serum T3 were normalized (similar to Slc16a2+/+ level) by p49 (Fig. 2G,H) which correlated with normalization of body weight of Slc16a2-/- (similar to Slc16a2+/+ rats) by p49 (Fig. 2E). These results may indicate an utility of rats as a model to study thyroid-related metabolism.

In support of transient reduced growth rate during early to late postnatal development, the dramatic reduction in testis size were found in Slc16a2-/- rats at p21, which was correlated with reduced seminiferous tubule size (Fig. 4A-D). This observation is in line with previous data where reduced testis size were caused by serum hyperthyroidism ${ }^{25,26}$. On the other hand, we found a larger testis of Slc16a2-/- rats at p49 with increased seminiferous tubule size (Fig. 4E-H). This effect was supported by the Sertoli cell numbers in different stage of development. As active thyroid hormone, T3 was found to play a key role in Sertoli cell cycle exit and differentiation ${ }^{8}$, local testis hypothyroidism due to Mct8 deficiency may prolonged Sertoli cell proliferation.

Other potential effect that alteration of TH may have is the development of the blood-testis barrier as literatures suggested that the expression of connexin 43 is regulated by $\mathrm{TH}^{27,28}$. Furthermore, hyperthyroidism was shown to elevate the number of Leydig cells ${ }^{29}$. Therefore, in future study, it will be interesting to investigate the blood-testis barrier and Leydig cells in Slc16a2-/- rats.

Other than testis development, we identified that Mct8 deficiency led to partially disrupted epididymal epithelium, which may suggest a potential role of TH in epididymal epithelial cell maintenance. We found the expression of Mct8 in epididymal epithelial cells and previous literature reported the expression of nuclear TH receptor (TR) in both cytosol and nucleus of rat epididymal epithelial cells. These suggest a potential role of TH in both genomic and non-genomic way in epididymal epithelial cells ${ }^{30}$. Therefore, in Mct8 deficient scenario, 
A

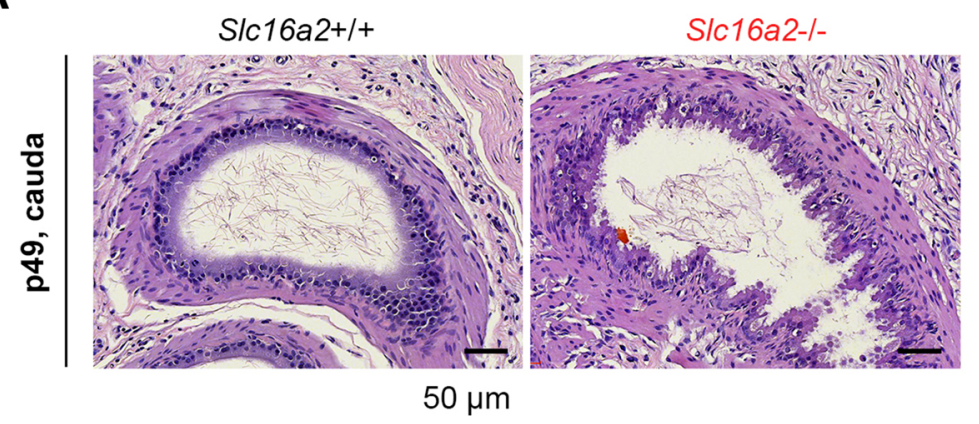

B

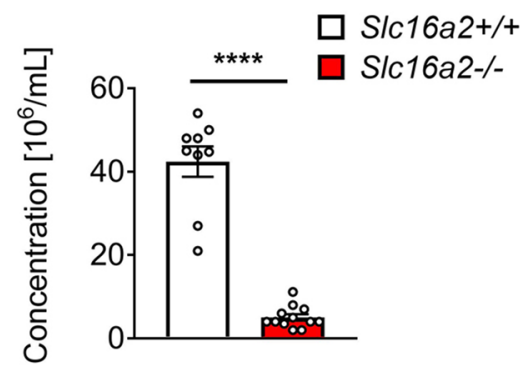

C

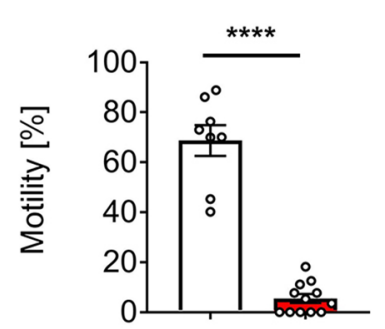

D

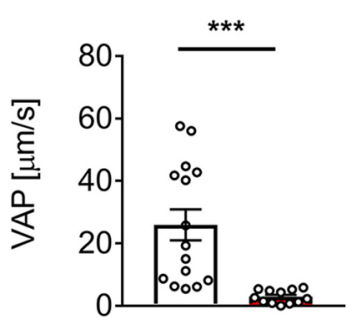

E

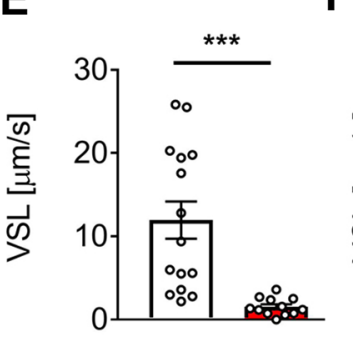

$\mathbf{F}$

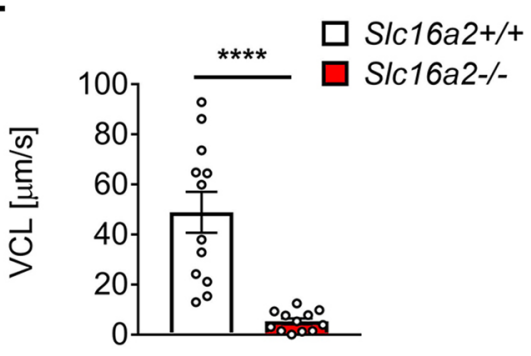

Figure 5. Altered spermiogenesis of Slc16a2-/- rat. (A) Representative images of H\&E stained cauda epididymis sections of Slc16a2+/+ and Slc16a2-/- rats at p49. Scale bar $=50 \mu \mathrm{m}$. (B-E) CASA analysis of spermatozoa of Slc16a2+/+ and Slc16a2-/- rats at p49. (B) Spermatozoa concentration; (C) spermatozoa motility; (D) spermatozoa average path velocity (VAP); (E) spermatozoa straight line velocity (VSL); (F) spermatozoa curvilinear velocity (VCL). $n=12, P<0.001$; $^{* * *} P<0.0001$; $^{* * * *}$.

there would be reduced TH transport into epididymal epithelial cells, which may have caused abnormal cellular physiology. Further studies are required to identify exact roles of TH on epididymal epithelial cells.

We have performed immunohistochemistry with anti-Mct8 antibody and found that Mct8 is expressed in epididymal epithelial cells (Supplementary Fig. 1). Epididymal spermatozoa seems also express Mct8 (Supplementary Fig. 1). As nuclear thyroid hormone receptor (TR) was found to be expressed on both cytosol and nucleus of rat epididymal epithelial cells, indicating a potential role of thyroid hormone in both genomic and non-genomic way ${ }^{30}$. Therefore, in Mct8 deficient scenario, there would be reduced thyroid hormone transport into epididymal epithelial cells which may caused normal cellular physiology.

As previous literatures suggested that thyroid hormones are required for proper functioning sperm cytoskeleton ${ }^{31-33}$, insufficient TH uptake by sperms in Slc16a2-/- rats would cause motility and shape abnormalities. Indeed, in our Slc16a2-/- rats, we found a reduction in number of sperms in epididymis and a significant changes in motility parameters from the CASA analysis. As alteration in TH levels in human were shown to adversely affect semen quality by compromising progressive sperm ${ }^{31}$, our results provide a direct evidence that alteration of TH levels by Mct8 deficiency affects sperm maturation in the epididymis.

Collectively, our results demonstrate that Mct8 plays an important role in TH action in the testis development and sperm maturation and motility. The Slc16a2-1- rat presented in this study can be a valuable model for studying the developmental and functional abnormalities in different tissues due to the alteration of THs, which only testis were partially explored in this study. As altered TH levels can affect other important cellular physiology as observed in patients with AHDS, it would be interesting to find out whether the present Slc16a2-I- rat model recapitulate any of these phenotypes.

\section{Methods}

Animals and ethics statement. Wild-type Sprague-Dawley (SD) female rats (7-13 weeks old) were purchased from Orient Bio (Korea). Rats were kept in animal facility (Seoul National University, Veterinary College). Standard water and food were supplied ad libitum (Daehan Bio Link). Animal room was climate controlled to provide temperatures of $22-25{ }^{\circ} \mathrm{C}$ on a $12 \mathrm{~h}$ light/dark cycle. All the experimental procedures as described below were performed in accordance with the IACUC guidance and regulations approved by the Seoul National University Institutional Animal Care and Use Committee (SNU-160719-2-7).

Generation of Slc16a2-/- rat. We generated Slc16a2 knockout rats by CRISPR/Cas9 genome editing. Genome Editor electroporator and LF501PT1-10 platinum plate electrode (length: $10 \mathrm{~mm}$, width: $3 \mathrm{~mm}$, height: $0.5 \mathrm{~mm}$, gap: $1 \mathrm{~mm}$ ) (BEX Co. Ltd., Tokyo, Japan) were used for electroporation.

The electrode was connected to the electroporator and was set under a stereoscopic microscope. 30-40 zygotes prepared by natural breeding (NB) electroporation at one time. The electroporation conditions were $30 \mathrm{~V}$ ( $3 \mathrm{~ms}$ 
$\mathrm{ON}+97 \mathrm{~ms}$ OFF $) \times 7$ times. Subsequently, the eggs were collected from the electrode chamber and subjected to four washes with M2 medium (Sigma) followed by four washes with mR1ECM medium(ARK Resource). After the incubation with $\mathrm{mR} 1 \mathrm{ECM}$ medium(ARK Resource) at $37^{\circ} \mathrm{C}$ and $5 \% \mathrm{CO} 2$, the eggs were allowed to develop to the two-cell stage and then transferred into pseudopregnant females.

Immunoblot analysis. Tissues were homogenized and lysed with Pierce RIPA buffer (25 mM Tris- $\mathrm{HCl}$ (pH 7.6), $150 \mathrm{mM} \mathrm{NaCl}, 1 \% \mathrm{NP}-40,1 \%$ sodium deoxycholate, $0.1 \%$ SDS, Thermo Scientific, Cat \# 89,900) for $30 \mathrm{~min}$ on ice. Tissue lysates were prepared by centrifugation $\left(13,000 \times \mathrm{rpm}\right.$ at $4{ }^{\circ} \mathrm{C}$ for $\left.30 \mathrm{~min}\right)$. Protein concentration was determined using Pierce BCA protein assay kit (Thermo Scientific, cat \# 23,225). Equal amounts of protein $(20 \mu \mathrm{g})$ were resolved on 4 to $15 \%$ SDS-PAGE and transferred to nitrocellulose membrane (Bio-Rad, Cat \# 1,620,115). After washing with TBS-T, the membranes were blocked with $2.5 \%$ skim milk for 30 min and incubated with appropriate primary antibodies Anti-MCT8 (Abcam, ab192828) and Anti- $\beta$-actin (Sigma, A2228). The membrane was washed then primary antibodies were detected with an HRP-conjugated secondary antibody.

qRT-PCR analysis. RNA was isolated from testis by using RNeasy Mini Kit (Qiagen) according to the manufacturer's instruction and treated with DNase-I to remove genomic DNA. The concentrations of total RNA were measured by Nanodrop ND-1000 spectrophotometer v3.7 (Thermo Specific) and complementary DNA (cDNA) was synthesized from $1 \mu \mathrm{g}$ of total RNA using High-Capacity cDNA Reverse Transcription Kit (Applied Biosystems) according to the manufacturer's instructions. Real-Time Quantitative Reverse Transcription Polymerase Chain Reaction (qRT-PCR) was performed with 5-20 ng of cDNA template, $2 \times$ Taqman Gene expression master mix and $20 \times$ Taqman probes (Life technologies) on ABI Prism 7900 HT Sequence detection system (Applied Biosystems). Gapdh served as an endogenous standard control. The qRT-PCR thermo-cycling reaction was 1 cycle at $50^{\circ} \mathrm{C}$ for $2 \mathrm{~min}, 1$ cycle at $95^{\circ} \mathrm{C}$ for $10 \mathrm{~min}$ and 40 cycles of $95^{\circ} \mathrm{C}$ for $15 \mathrm{~s}$, then $60^{\circ} \mathrm{C}$ for $1 \mathrm{~min}$.

Accession number of Taqman probes used for qRT-PCR.

\begin{tabular}{|l|l|}
\hline Target Gene & Taqman Gene Expression Assay \\
\hline Gapdh & Rn01775763_g1 \\
\hline Slc16a2 & Rn00596041_m1 \\
\hline Slco1c1 & Rn00584891_m1 \\
\hline Slc7a5 & Rn00569313_m1 \\
\hline Slc7a8 & Rn00584909_m1 \\
\hline Prm1 & Rn02345725_g1 \\
\hline
\end{tabular}

Measurement of serum TH, Testosterone. Blood was collected in SST (Serum separate tube) tubes by abdominal vein puncture and immediately centrifuged at 3,000 rpm for $10 \mathrm{~min}$. The serum samples were stored at $-80^{\circ} \mathrm{C}$ until further analysis. T3, T4 and TSH concentrations in serum were measured using an electrochemiluminescence immunoassay (Elecsys, Roche, Germany). Testosterone concentrations in serum were measured using an Enzyme-Linked Immunosorbent Assay (Testosterone Rat/Mouse ELISA, Calbiotech, USA).

Histology. Testes were fixed in Bouin's solution for $2 \mathrm{~h}$ (fetal and neonatal testes) or overnight (adult testes), briefly washed in $70 \%$ ethanol, cut transversally in 2 halves and paraffin embedded for sectioning at the cut area. Hematoxylin and eosin (H\&E) staining, as well as immunohistochemistry staining for the The diameter of a seminiferous tubule was defined as the shortest distance between two parallel tangent lines of the outer edge of the tubule. Testis sections of 4 mice/group were obtained by optical microscopy using an Olympus IX51 camera $\times 10$-microm eyepiece coupled with an $\times 10$ objective glass (Olympus Japan). The seminiferous tubule diameter was measured using the QCapture Pro software (version 6.0; QImaging). 200 tubules were measured per animal.

Immunohistochemical staining was done using UltraVision LP Large Volume Detection System (Thermo Scientific) according to the manufacturer's procedure. Briefly, $5-\mu \mathrm{m}$ thick sections were deparaffinized in xylene, and rehydrated in decreasing ethanol concentrations. After antigen retrieval with Proteinase K, the sections were incubated with blocking solution (Hydrogen Peroxide Block) for $10 \mathrm{~min}$ at room temperature (RT). The primary antibody Anti-MCT8 (MBL, BMP031) were applied and incubated for overnight at $4{ }^{\circ} \mathrm{C}$. After washing the primary antibody, the slides were incubated with Primary Antibody Enhancer for 20 min at RT, followed by incubation with HRP Polymer for $30 \mathrm{~min}$ at RT. The sections were developed using DAB (DAB + Substrate Chromogen System, DAKO) and then were counterstained with Mayer's hematoxylin.

In vitro fertilization (IVF). Sprague Dawley female rats (4-weeks-old) were induced to superovulate using intraperitoneal injections of PMSG on Day 1 and hCG on Day 3. On Day 4, male rats were sacrificed and the cauda epididymis were quickly removed, allowing the sperm capacitates to place in the incubator for $1 \mathrm{~h}$ before insemination. During this period, the superovulated female rat was sacrificed and the ampullae were quickly removed. To collect cumulus-oocyte-complex (COC), the ampullae were placed in a fertilization dish (FERTIUP PM 0.5ML-CARD MEDIUM set, KYD-005-EX). The sperm added into the fertilization dish included the COC or cumulus-free oocytes using a pipette. The fertilization dish was incubated for approximately $3 \mathrm{~h}$ at $37^{\circ} \mathrm{C}$. After this incubation, embryo were removed from the fertilization dish and washed 3 times with the HTF 
(ARK Resource Co., Ltd.). The HTF drops that covered with paraffin oil incubated for overnight at $37^{\circ} \mathrm{C}$ and the number of two-cell was counted on Day 5.

CASA. Mature male rats were sacrificed and the two epididymis were removed aseptically. Left and right epididymis were transferred to the $10-12 \mathrm{ml}$ liquid paraffin in a $60 \mathrm{~mm}$ petri dish. A small incision around $0.5 \mathrm{~mm}$ was made in the caudal epididymis and 1-2 drop of sperms were transferred to pre-warmed M2 media to dilute 1:500 and maintained at $37^{\circ} \mathrm{C}$ for $3 \mathrm{~min}$. During this time, the dish was rotated every minute to disperse sperm. Thirty microliter sperms near the marginal drop were collected and loaded on standard count 2-chamber slides (Leja, SC 100-01-02-B). Sperm motility was analyzed by CASA and images were taken by Olympus BX 50. Samples were analysed three times at different sites.

Statistical analysis. The GraphPad Prism software for statistical analysis. Unless stated otherwise, comparisons between more than 2 experimental groups were performed by ANOVA and Tukey's post hoc test, whereas statistical significance between 2 groups was determined by the Student's $t$ test. $P$ value smaller than 0.05 was considered statistically significant.

\section{Data availability}

The raw data presented in this manuscript is available upon request to JYL (jy.lee2@toolgen.com).

Received: 7 June 2019; Accepted: 7 June 2020

Published online: 07 July 2020

\section{References}

1. Cheng, S. Y., Leonard, J. L. \& Davis, P. J. Molecular aspects of thyroid hormone actions. Endocr. Rev. 31, 139-170. https://doi. org/10.1210/er.2009-0007 (2010).

2. Williams, G. R. Neurodevelopmental and neurophysiological actions of thyroid hormone. J. Neuroendocrinol. 20, 784-794. https ://doi.org/10.1111/j.1365-2826.2008.01733.x (2008).

3. Bernal, J., Guadano-Ferraz, A. \& Morte, B. Thyroid hormone transporters-functions and clinical implications. Nat. Rev. Endocrinol. 11, 690. https://doi.org/10.1038/nrendo.2015.186 (2015).

4. Friesema, E. C. et al. Identification of monocarboxylate transporter 8 as a specific thyroid hormone transporter. J. Biol. Chem. 278, 40128-40135. https://doi.org/10.1074/jbc.M300909200 (2003).

5. Hernandez, A. Thyroid hormone role and economy in the developing testis. Vitam. Horm. 106, 473-500. https://doi.org/10.1016/ bs.vh.2017.06.005 (2018).

6. Buzzard, J. J., Morrison, J. R., O’Bryan, M. K., Song, Q. \& Wreford, N. G. Developmental expression of thyroid hormone receptors in the rat testis. Biol. Reprod. 62, 664-669. https://doi.org/10.1095/biolreprod62.3.664 (2000).

7. Jannini, E. A. et al. Ontogenetic pattern of thyroid hormone receptor expression in the human testis. J. Clin. Endocrinol. Metab. 85, 3453-3457. https://doi.org/10.1210/jcem.85.9.6803 (2000).

8. Gao, Y., Lee, W. M. \& Cheng, C. Y. Thyroid hormone function in the rat testis. Front. Endocrinol. (Lausanne) 5, 188. https://doi. org/10.3389/fendo.2014.00188 (2014).

9. Schwartz, C. E. et al. Allan-Herndon-Dudley syndrome and the monocarboxylate transporter 8 (MCT8) gene. Am. J. Hum. Genet. 77, 41-53. https://doi.org/10.1086/431313 (2005).

10. Ono, E. et al. Three novel mutations of the MCT8 (SLC16A2) gene: individual and temporal variations of endocrinological and radiological features. Clin. Pediatr. Endocrinol. 25, 23-35. https://doi.org/10.1297/cpe.25.23 (2016).

11. Dumitrescu, A. M., Liao, X. H., Weiss, R. E., Millen, K. \& Refetoff, S. Tissue-specific thyroid hormone deprivation and excess in monocarboxylate transporter (mct) 8-deficient mice. Endocrinology 147, 4036-4043. https://doi.org/10.1210/en.2006-0390 (2006).

12. Trajkovic, M. et al. Abnormal thyroid hormone metabolism in mice lacking the monocarboxylate transporter 8. J. Clin. Invest. 117, 627-635. https://doi.org/10.1172/JCI28253 (2007).

13. Jacob, H. J. Functional genomics and rat models. Genome Res. 9, 1013-1016 (1999).

14. Xu, S. et al. The 14th Ile residue is essential for Leptin function in regulating energy homeostasis in rat. Sci. Rep. 6, 28508. https:// doi.org/10.1038/srep28508 (2016).

15. Hagenbuch, B. \& Meier, P. J. The superfamily of organic anion transporting polypeptides. Biochim. Biophys. Acta 1609, 1-18 (2003).

16. Martinez, M. E. et al. The type 3 deiodinase is a critical determinant of appropriate thyroid hormone action in the developing testis. Endocrinology 157, 1276-1288. https://doi.org/10.1210/en.2015-1910 (2016).

17. Kinne, A., Schulein, R. \& Krause, G. Primary and secondary thyroid hormone transporters. Thyroid Res. 4(Suppl 1), S7. https:// doi.org/10.1186/1756-6614-4-S1-S7 (2011).

18. Muller, J. et al. Tissue-specific alterations in thyroid hormone homeostasis in combined Mct10 and Mct8 deficiency. Endocrinology 155, 315-325. https://doi.org/10.1210/en.2013-1800 (2014).

19. Trajkovic-Arsic, M. et al. Impact of monocarboxylate transporter-8 deficiency on the hypothalamus-pituitary-thyroid axis in mice. Endocrinology 151, 5053-5062. https://doi.org/10.1210/en.2010-0593 (2010).

20. Hernandez, A. Thyroid hormone deiodination and action in the gonads. Curr. Opin. Endocr. Metab. Res. 2, 18-23. https://doi. org/10.1016/j.coemr.2018.01.010 (2018).

21. Aumuller, G., Steinbruck, M., Krause, W. \& Wagner, H. J. Distribution of vimentin-type intermediate filaments in Sertoli cells of the human testis, normal and pathologic. Anat. Embryol. (Berl) 178, 129-136. https://doi.org/10.1007/bf02463646 (1988).

22. Gibbs, R. A. et al. Genome sequence of the Brown Norway rat yields insights into mammalian evolution. Nature 428, 493-521. https://doi.org/10.1038/nature02426 (2004).

23. Iannaccone, P. M. \& Jacob, H. J. Rats! Dis. Model Mech. 2, 206-210. https://doi.org/10.1242/dmm.002733 (2009).

24. Ellenbroek, B. \& Youn, J. Rodent models in neuroscience research: is it a rat race?. Dis. Model Mech. 9, 1079-1087. https://doi. org/10.1242/dmm.026120(2016).

25. Holsberger, D. R., Kiesewetter, S. E. \& Cooke, P. S. Regulation of neonatal Sertoli cell development by thyroid hormone receptor alpha1. Biol. Reprod. 73, 396-403. https://doi.org/10.1095/biolreprod.105.041426 (2005).

26. Bakke, J. L., Lawrence, N. L., Bennett, J. \& Robinson, S. The late effects of neonatal hyperthyroidism upon the feedback regulation of TSH secretion in rats. Endocrinology 97, 659-664. https://doi.org/10.1210/endo-97-3-659 (1975).

27. Marchlewska, K. et al. The long-term effects of FSH and triiodothyronine administration during the pubertal period on Connexin 43 expression and spermatogenesis efficiency in adult rats. J. Exp. Zool. A Ecol. Genet. Physiol. 323, 256-265. https://doi. org/10.1002/jez.1919 (2015). 
28. Marchlewska, K. et al. Maturational changes in connexin 43 expression in the seminiferous tubules may depend on thyroid hormone action. Arch. Med. Sci. 9, 139-145. https://doi.org/10.5114/aoms.2013.33074 (2013).

29. Fadlalla, M. B. et al. Effects of hyper- and hypothyroidism on the development and proliferation of testicular cells in prepubertal rats. Anim. Sci. J. 88, 1943-1954. https://doi.org/10.1111/asj.12883 (2017).

30. De Paul, A. L. et al. Thyroid hormone receptor alpha 1-beta 1 expression in epididymal epithelium from euthyroid and hypothyroid rats. Histochem. Cell. Biol. 129, 631-642. https://doi.org/10.1007/s00418-008-0397-8 (2008).

31. Corrales Hernandez, J. J. \& Miralles Garcia, J. M. Garcia Diez LC (1990) Primary hypothyroidism and human spermatogenesis. Arch. Androl. 25, 21-27. https://doi.org/10.3109/01485019008987590 (1990).

32. Zamoner, A. et al. Propylthiouracil-induced congenital hypothyroidism upregulates vimentin phosphorylation and depletes antioxidant defenses in immature rat testis. J. Mol. Endocrinol. 40, 125-135. https://doi.org/10.1677/JME-07-0089 (2008).

33. Condorelli, R. A. et al. (2019) Thyroid hormones and spermatozoa: in vitro effects on sperm mitochondria, viability and dna integrity. J. Clin. Med 8, 15-20. https://doi.org/10.3390/jcm8050756 (2019).

\section{Acknowledgements}

This work was partially supported by grants from the Korean Health Technology R\&D Project, Ministry of Health \& Welfare (HI16C0426) and by NRF grants funded by the Korean government (2017M3A9B4061404 and 2018M3A9H3020844).

\section{Author contributions}

H.S.B., Y.J. generated and maintained knockout rats. H.S.B., Y.J., H.S.W., H.K.K., H.S., G.C., K.J.L., H.L. and K.K. performed experiments. O.K., G.J. and J.Y.L. designed experiments. G.J., J.M.L. and J.Y.L. analyzed the data. J.Y.L. wrote the manuscript and prepared figures.

\section{Competing interests}

The authors declare no competing interests.

\section{Additional information}

Supplementary information is available for this paper at https://doi.org/10.1038/s41598-020-67594-2.

Correspondence and requests for materials should be addressed to J.Y.L.

Reprints and permissions information is available at www.nature.com/reprints.

Publisher's note Springer Nature remains neutral with regard to jurisdictional claims in published maps and institutional affiliations.

(c) (i) Open Access This article is licensed under a Creative Commons Attribution 4.0 International License, which permits use, sharing, adaptation, distribution and reproduction in any medium or format, as long as you give appropriate credit to the original author(s) and the source, provide a link to the Creative Commons license, and indicate if changes were made. The images or other third-party material in this article is included in the article's Creative Commons license, unless indicated otherwise in a credit line to the material. If material is not included in the article's Creative Commons license and your intended use is not permitted by statutory regulation or exceeds the permitted use, you will need to obtain permission directly from the copyright holder. To view a copy of this license, visit http://creativecommons.org/licenses/by/4.0/.

(C) The Author(s) 2020 